\title{
Plásticos no ambiente marinho frio: uma revisão sobre o potencial de biodegradação
}

\section{microbiana}

\author{
Plastics in the cold marine environment: a review of the potential for microbial biodegradation \\ Plásticos en el medio marino frío: una revisión del potencial de biodegradación microbiana
}

Recebido: 07/03/2021 | Revisado: 14/03/2021 | Aceito: 17/03/2021 | Publicado: 20/03/2021

\author{
Linda Rocio Ojeda Almeida \\ ORCID: https://orcid.org/0000-0001-9865-582X \\ Universidade Federal da Integração Latino-Americana, Brasil \\ E-mail: linda.almeida@aluno.unila.edu.br \\ Júlia Ronzella Ottoni \\ ORCID: https://orcid.org/0000-0003-1229-7676 \\ Universidade Federal da Integração Latino-Americana, Brasil \\ E-mail: julia.ottoni@unila.edu.br \\ Michel Rodrigo Zambrano Passarini \\ ORCID: https://orcid.org/0000-0002-8614-1896 \\ Universidade Federal da Integração Latino-Americana, Brasil \\ E-mail: michel.passarini@unila.edu.br
}

\begin{abstract}
Resumo
Os plásticos são produtos amplamente utilizados pois apresentam propriedades desejáveis para diversos setores industriais, como durabilidade, plasticidade e transparência. Toneladas deste material vêm se acumulando em diversos ambientes do mundo, tornando-se uma problemática ambiental. Uma alternativa de eliminação deste material é a busca por microrganismos adaptados ao frio, os quais desenvolvem vias catalíticas diferenciadas para se adaptarem às baixas temperaturas, podendo sintetizar enzimas aptas a metabolizar estes materiais. Devido à ausência ou baixa atividade destas enzimas, essa estratégia ainda é um desafio sem solução efetiva. O objetivo da presente revisão foi avaliar as pesquisas sobre a biodegradação dos plásticos por microrganismos de ambientes marinhos frios, nos últimos 10 anos, utilizando-se os seguintes descritores: "Plastic biodegradation AND cold oceans", "Plastic biodegradation AND (Psychrophile OR Psychrophilic)", "PETases AND (bacteria OR fungus)" e "Extremophile AND plastic biodegradation". Foi realizado um levantamento de publicações nas bases científicas Pubmed, Scopus e na ferramenta de busca Google Scholar. Foram encontrados 11.481 trabalhos, dos quais $97.9 \%, 1.84 \%$ e $0.26 \%$ foram recuperados do Google Scholar, Scopus e Pubmed, respectivamente. Espécies pertencentes aos gêneros Streptomyces, Corynebacterium, Arthrobacter, Micrococcus, Pseudomonas e Rhodococcus foram as mais frequentemente mencionadas como potenciais degradadores de plásticos em ambientes marinhos frios. Os resultados deste trabalho mostram que os estudos voltados para a degradação de plásticos por microrganismos de ambiente frio ainda são escassos, abrindo assim, perspectivas para que novos trabalhos sejam realizados abordando essa temática.
\end{abstract}

Palavras-chave: Plástico; Oceano; Ambiente extremo; Biorremediação; Psicrofílicos.

\begin{abstract}
Plastics are widely used products due to their desirable properties for several industrial sectors, such as durability, plasticity, and transparency. Tons of this material has been accumulating in different environments around the world, making it an environmental problem. An alternative to eliminating this material is the search for microorganisms adapted to the cold, which develop different catalytic pathways to adapt to low temperatures, being able to synthesize enzymes capable of metabolizing these materials. Due to the absence or low activity of these enzymes, this strategy is still a challenge with no effective solution. The purpose of this review was to evaluate research on the biodegradation of plastics by microorganisms from cold marine environments, in the last 10 years, using the following descriptors: "Plastic biodegradation AND cold oceans", "Plastic biodegradation AND (Psychrophile OR Psychrophilic)", "PETases AND (bacteria OR fungus)" and "Extremophile AND plastic biodegradation". A survey of publications was carried out on the scientific bases Pubmed, Scopus, and on the Google Scholar search tool. About 11.481 works were found, of which 97.9\%, 1.84\%, and 0.26\% were retrieved from Google Scholar, Scopus, and Pubmed, respectively. Species belonging to the genera Streptomyces, Corynebacterium, Arthrobacter, Micrococcus, Pseudomonas, and Rhodococcus were the most frequently mentioned as potential degraders of plastics in cold marine environments. The results of this work show that studies focused on the degradation of plastics by microorganisms in a cold environment are still scarce, thus opening perspectives for new studies to be carried out addressing this theme.
\end{abstract}

Keywords: Plastic; Ocean; Extreme environment; Bioremediation; Psychrophilic. 


\begin{abstract}
Resumen
Los plásticos son productos muy utilizados porque tienen propiedades deseables para varios sectores industriales, como durabilidad, plasticidad y transparencia. Toneladas de este material se han ido acumulando en diferentes entornos de todo el mundo, lo que lo convierte en un problema medioambiental. Una alternativa para la eliminación de este material es la búsqueda de microorganismos adaptados al frío, que desarrollen vías catalíticas diferenciadas para adaptarse a las bajas temperaturas, pudiendo sintetizar enzimas capaces de metabolizar estos materiales. Debido a la ausencia o baja actividad de estas enzimas, esta estrategia sigue siendo un desafío sin una solución eficaz. El propósito de esta revisión fue evaluar la investigación sobre la biodegradación de plásticos por microorganismos de ambientes marinos fríos, en los últimos 10 años, utilizando los siguientes descriptores: "Biodegradación de plásticos $Y$ océanos fríos", "Biodegradación de plásticos $Y$ (psicrófilos o psicrófilos)", "PETasas $Y$ (bacterias u hongos)" y "Biodegradación de plásticos y extremófilos". Se realizó un relevamiento de publicaciones en las bases científicas Pubmed, Scopus y en la herramienta de búsqueda Google Scholar. Se encontraron 11.481 trabajos, de los cuales $97.9 \%, 1.84 \%$ y $0.26 \%$ se recuperaron de Google Scholar, Scopus y Pubmed, respectivamente. Las especies pertenecientes a los géneros Streptomyces, Corynebacterium, Arthrobacter, Micrococcus, Pseudomonas y Rhodococcus fueron las más mencionadas como degradantes potenciales de plásticos en ambientes marinos fríos. Los resultados de este trabajo muestran que aún son escasos los estudios sobre la degradación de plásticos por microorganismos en un ambiente frío, lo que abre perspectivas para nuevos estudios a realizar sobre este tema.
\end{abstract}

Palabras clave: Plástico; Océano; Ambiente extremo; Biorremediación; Psicrófilo.

\title{
1. Introdução
}

Apesar de os plásticos serem utilizados há um século, a produção industrial deste produto teve início apenas em 1950 (Geyer et al., 2017). Até 2015, o mundo já havia produzido cerca de 7,8 bilhões de toneladas de plástico (Ritchie, 2018). Estimase a existência de no mínimo 5,25 trilhões de partículas de plástico menores de $5 \mathrm{~mm}$ (microplásticos), pesando 268.940 toneladas, flutuando nos oceanos do mundo (Eriksen et al., 2014). Entretanto, previsões apontam que a produção deverá ser dobrada nos próximos 20 anos, podendo ser até quatro vezes maior até o final de 2050 (Ellen MacArthur Foundation, 2016). Os plásticos, ou materiais poliméricos ou polímeros capazes de adquirirem condições plásticas por ação do calor e pressão, são classificados de acordo com o material polimérico, incluindo: tereftalato de polietileno (PET), polietileno (PE), polietileno de alta densidade (HDPE), polietileno de baixa densidade (LDPE), cloreto de polivinil (PVC), polipropileno (PP), poliestireno (PS) e plásticos diversos, que incluem policarbonato, poliláctico, fibra de vidro, nylon, entre outros (Urbanek et al., 2018). Estes materiais acabam indo para o oceano de diversas maneiras, incluindo má gestão e descarte inadequado nos esgotos, operações de aterros costeiros e lixo transportado em córregos e rios. Aproximadamente, $80 \%$ dos plásticos que se encontram nos oceanos são de origem terrestre, e os demais $20 \%$ são gerados pelo turismo, pesca, indústrias e navios/embarcações (Mattsson et al., 2015; Veiga et al., 2016).

No ambiente marinho, os plásticos mediantes fatores abióticos como radiação UV, oxigênio, temperatura e estresse físico (Geweret et al., 2015), são fragmentados, formando detritos de plástico (microplásticos), que acabam adentrando em giros subtropicais, os quais podem chegar a um milhão de $\mathrm{km}^{2}$. Os detritos plásticos prejudicam animais como peixes, tartarugas marinhas, pássaros e mamíferos marinhos, devido a ingestão e emaranhamento marinho (Gregory, 2009). A quantidade de plásticos observada flutuando no oceano aberto representa apenas uma porção do total. Mais de dois terços do lixo plástico acabam no fundo do mar, com metade do restante sendo levado até as praias e a outra metade flutuando na superfície ou abaixo dela (Andrady, 2011).

Os plásticos no ambiente marinho são revestidos por matéria orgânica e inorgânica, o que é conhecido como "filme de condicionamento". Este filme serve como fonte de carbono e nutrientes para microrganismos, os quais colonizam rapidamente sua superfície, criando um biofilme. A diversidade e abundância dos microrganismos nos ambientes marinhos é muito extensa, podendo atingir até centenas de milhões de células bacterianas em um grama de sedimento marinho (Harrison et al., 2011). Comunidades que habitam ambientes frios vêm desenvolvendo capacidades metabólicas únicas, as quais são responsáveis pela adaptação celular em ambientes que apresentam baixas temperaturas, disponibilidade limitada de nutrientes e alta salinidade, 
condições estas que exigem adaptações estruturais e funcionais para a sobrevivência (Margesin \& Miteva, 2011). Desta maneira, os microrganismos psicrofílicos e/ou psicrotolerantes vêm despertando interesse das pesquisas voltadas à bioprospecção para a exploração deste arsenal enzimático diferenciado dos microrganismos terrestres e/ou mesofílicos (Ottoni et al., 2020; Yang et al., 2019).

Diversas linhagens microbianas incluindo bactérias e fungos foram identificadas em estudos utilizando amostras plásticas do mar ou de plásticos testados experimentalmente em condições marinhas (Jacquin et al., 2019). Assim, podemos citar os gêneros Brevibacillus, Bacillus, Comamonas, Stenotrophomonas, Clostridium, Ralstonia, Enterobacter, Pseudomonas, Acinetobacter; Aspergillus, Penicillium, Fusarium (Ingavale \& Raut, 2018; Kumaravel et al., 2010; Kyaw et al., 2012; MartínezTobón et al., 2018; Nimchua et al., 2007; Peixoto et al., 2017; Pramila, 2012; Sheik et al., 2015, Sowmya et al., 2012; Yang et al., 2014).

Para que a biodegradação ocorra é necessário que a degradação abiótica (física e química) do plástico esteja presente, o que leva ao enfraquecimento do polímero (İpekoglu et al., 2007). Esse processo abiótico pode ocorrer, mediante fatores como luz solar, calor, umidade e condições químicas capazes de quebrar a cadeia polimérica. Dussud \& Ghiglione (2014), descreveram a biodegradação microbiana do plástico, que consiste em quatro etapas: $i$ ) biodeterioração: microrganismos que formam um biofilme, secretam substâncias poliméricas extracelulares (EPS) e ocorre adesão à superfície plástica (coesão). Estes EPS se acumulam entre fissuras e poros causados pelos fatores abióticos, aumentando o rompimento das ligações químicas e dos poros. Assim, ocorre a formação de compostos ácidos, incluindo ácido nitroso, nítrico ou sulfúrico, liberados por bactérias quimiolitotróficas, bem como ácidos orgânicos produzidos por comunidades quimiorganotróficas, promovendo a degradação progressiva da matriz plástica; ii) biofragmentação: nesta etapa o polímero é quebrado em oligo e monômeros, pelas enzimas oxigenases, lipases e esterases, finalizando a quebra do polímero. Estas reações se dão principalmente nas extremidades do polímero (fissuras e poros); iii) assimilação: ocorre pela internalização dos monômeros de plástico por transportadores específicos ou por passagem passiva dos monômeros pela parede e/ou membrana celular; $i v$ ) mineralização: uma vez internalizado, a degradação dos monômeros ocorre por oxidação, de acordo com três vias metabólicas possíveis, incluindo respiração aeróbia, anaeróbica ou fermentação, dependendo do grupo microbiano. Os metabólitos secretados oxidados incluem $\mathrm{CO}_{2}, \mathrm{~N}_{2}, \mathrm{CH}_{4}$ e $\mathrm{H}_{2} \mathrm{O}$ (Figura 1).

Figura 1 - As diferentes etapas da biodegradação do plástico por microrganismos. Fonte: Dussud and Ghiglione (2014), modificado.

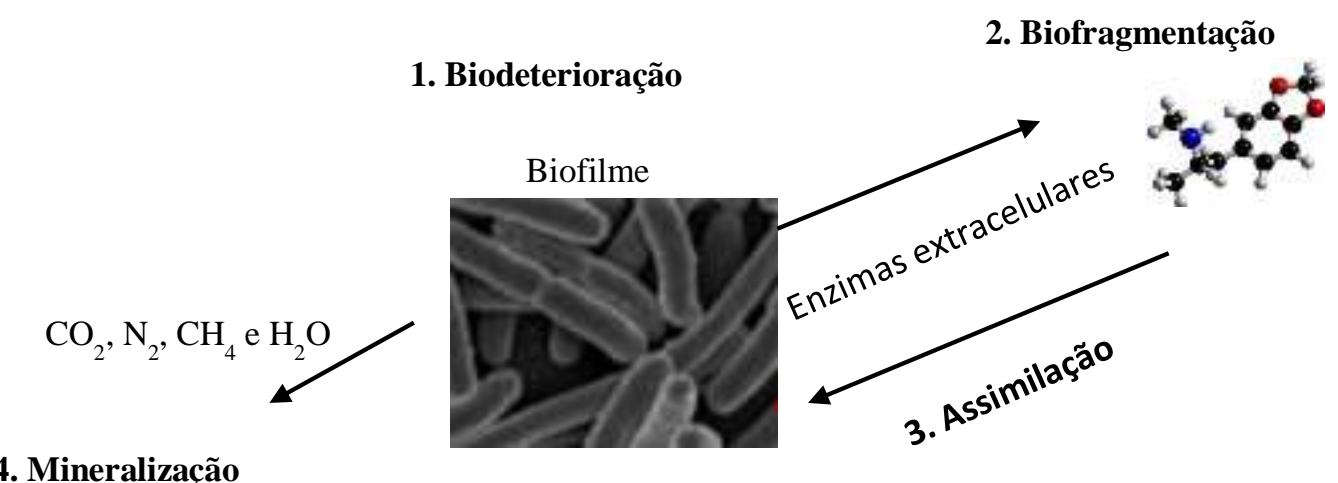

\section{Mineralização}

Fonte: Autores.

Nos últimos anos, as pesquisas vêm explorando ambientes extremos e pouco explorados, incluindo regiões dos polos, habitats marinhos complexos e ambientes hipertermais. Estas investigações apresentam como principais objetivos a descoberta de novos compostos bioativos de interesse bem como sua possível aplicação em processos industriais e na área da saúde (Mehetre 
et al., 2018; Undabarrena et al., 2016; Yogabaanu et al., 2017). No sistema marinho Antártico (também conhecido como Oceano Austral), detritos de plástico são encontrados tanto na superfície como nos sedimentos do fundo do mar. Nessas regiões, os microplásticos foram encontrados em abundância, com valores entre 16 e 766 partículas sintéticas por $\mathrm{m}^{2}$ (Waller et al. 2017).

Muitas linhagens marinhas da espécie Pseudomonas pertucinogena são adaptadas a ambientes frios, com relatos de crescimento a temperaturas abaixo de $15{ }^{\circ} \mathrm{C}$ e com tolerância a concentrações moderadas de sal. Algumas das espécies descritas vivem em associação com plantas aquáticas e esponjas, em uma relação simbiótica. Além de colonizar esses substratos, os ambientes contaminados parecem ser um dos habitats para essas bactérias marinhas (Lamendella et al., 2014). As enzimas produzidas por bactérias da linhagem $P$. pertucinogena foram mencionadas em alguns estudos, principalmente em trabalhos de degradação de polímeros e síntese de moléculas quirais (Haernvall et al., 2017; Haernvall et al., 2018). O potencial de P. pelagia em degradar efetivamente poliésteres à base de ácido ftálico iônico foi demonstrado recentemente por Haernvall et al., (2017) em um estudo no qual a enzima biocatalisadora responsável pela degradação foi uma lipase.

Desta forma, esta revisão foi realizada utilizando trabalhos científicos reportados nos últimos 10 anos, os quais relataram a utilização de microrganismos derivados de ambientes marinhos frios, empregados em processos de degradação e/ou assimilação ou até mesmo compreensão do metabolismo microbiano relacionado aos plásticos.

\section{Metodologia}

Para a realização desta revisão, foi empregada uma metodologia analítico-exploratória qualitativa (Pereira et al., (2018; Camargo et al., 2020) em bases de dados digitais de artigos científicos incluindo Pubmed, Scopus e a ferramenta de busca Google Scholar. Foram considerados os dados no intervalo de tempo de 11/2010 até 11/2020, da seguinte maneira:

Para o levantamento dos conteúdos nas bases de dados, foi utilizado uma sequência de palavras-chave atribuídas pelos autores a partir dos objetivos da pesquisa. Assim, os descritores utilizados foram: "Plastic biodegradation AND cold oceans", "Plastic biodegradation AND (Psychrophile OR Psychrophilic)", "PETases AND (bacteria OR fungus)" e "Extremophile AND plastic biodegradation".

Após a definição dos termos de busca, foram aplicados filtros para limitar a curva temporal entre os anos de 2010 a 2020, bem como a exclusão dos resultados levantados de publicações do tipo monografias, trabalhos de conclusão de curso, dissertações de mestrado, teses de doutorado, publicações em congressos e anais de eventos. Foi proposto a realização de um corte de periódicos com fator de impacto baixos, sendo assim, consideramos para a realização deste trabalho, índices de fatores de impactos (JCR - Journal Citation Reports), acima de 2,0, bem como a escolha das áreas temáticas de pesquisa "Ciência Ambiental", "Engenharia Química e Bioquímica" e "Genética e Biologia Molecular" (as demais áreas, apresentavam trabalhos irrelevantes para o presente estudo). As informações das publicações científicas foram compiladas em tabelas, nas quais foram relacionadas a quantidade de publicações obtidas com as palavras-chaves bem como a quantidade final após a realização dos cortes.

\section{Resultados e Discussão}

Após busca dos trabalhos publicados nos períodos definido, foram encontrados 11.481 trabalhos científicos nas bases de dados e na ferramenta de busca utilizadas. Em seguida, foi realizada uma seleção dos trabalhos em função dos filtros propostos nesta metodologia incluindo curva temporal entre os anos de 2010 a 2020, índices de fatores de impactos (JCR acima de 2,0), o que reduziu o número de trabalhos para 105 (Tabela 1). 
Tabela 1 - Artigos científicos encontrados com os descritores nas bases de dados Scopus, NCBI e Google Scholar.

\begin{tabular}{|c|c|c|c|c|}
\hline Descritores & Scopus & Pubmed & Google Scholar & $\operatorname{JCR}(>2,0)$ \\
\hline $\begin{array}{l}\text { Plastic biodegradation AND cold } \\
\text { oceans }\end{array}$ & 158 & 12 & 7000 & 24 \\
\hline $\begin{array}{c}\text { Plastic biodegradation AND } \\
\text { (Psychrophile OR Psychrophilic) }\end{array}$ & 30 & 3 & 2080 & 35 \\
\hline $\begin{array}{c}\text { PETases AND (bacteria OR } \\
\text { fungus) }\end{array}$ & 10 & 1 & 40 & 22 \\
\hline $\begin{array}{l}\text { Extremophile AND plastic } \\
\text { biodegradation }\end{array}$ & 13 & 14 & 2120 & 24 \\
\hline TOTAL & 211 & 30 & 11240 & 105 \\
\hline
\end{tabular}

Fonte: Autores.

Observando a Tabela 1, podemos verificar que a busca utilizando o Google Scholar retornou uma maior quantidade de trabalhos $(\mathrm{n}=11.240)$, seguido pelo Scopus e Pubmed, $\mathrm{n}=211$ e 30, respectivamente. A quantidade bem superior encontrada no Google Scholar, pode ser justificada pela inespecificidade da busca nessa plataforma, abrangendo todo tipo de pesquisa nas áreas avaliadas. Levando em consideração todos os filtros utilizados neste estudo, a Tabela 2 foi elaborada com o objetivo de apontar os 5 artigos científicos mais relevantes, para cada descritor utilizado. Os dados da Tabela mostram resumidamente os pontos: ano de publicação, periódico, JCR, autores, linhagens utilizadas no estudo e o(s) banco(s) de dados e/ou ferramenta de busca onde o trabalho foi encontrado.

Tabela 2 - Lista dos 5 artigos mais relevantes para cada descritor utilizado nesta revisão.

\begin{tabular}{|c|c|c|c|c|c|}
\hline $\begin{array}{c}\text { Ano de } \\
\text { publicação }\end{array}$ & Periódico & JCR & Autores & Gênero/espécie estudada & Banco de dados \\
\hline \multicolumn{6}{|c|}{ "Plastic biodegradation AND cold oceans" } \\
\hline 2017 & $\begin{array}{l}\text { Science of the Total } \\
\text { Environment }\end{array}$ & 6.55 & Paço et al. & Zalerion maritimum & \\
\hline 2013 & $\begin{array}{l}\text { Marine Pollution } \\
\text { Bulletin }\end{array}$ & 4.05 & $\begin{array}{l}\text { Harshvardhan } \\
\text { \& Bhavanath }\end{array}$ & $\begin{array}{c}\text { Kocuria palustris M16, Bacillus } \\
\text { pumilus M27 e Bacillus subtilis } \\
\text { H1584 }\end{array}$ & $\begin{array}{c}\text { Google Scholar e } \\
\text { Scopus }\end{array}$ \\
\hline 2020 & $\begin{array}{l}\text { Marine } \\
\text { Environment } \\
\text { Research }\end{array}$ & 2.7 & $\begin{array}{l}\text { Giacomucci et } \\
\text { al. }\end{array}$ & $\begin{array}{c}\text { Clostridium, Acetobacterium, } \\
\text { Dethiosulfovibrio. e } \\
\text { Sporobacter }\end{array}$ & \\
\hline 2010 & $\begin{array}{l}\text { Jornal of japanese } \\
\text { Society for } \\
\text { Extremophiles }\end{array}$ & 2.57 & Sekiguchi et al. & $\begin{array}{c}\text { Shewanella, Moritella, } \\
\text { Psychrobacter e Pseudomonas }\end{array}$ & Google Scholar \\
\hline 2019 & $\begin{array}{l}\text { Marine Pollution } \\
\text { Bulletin }\end{array}$ & 4.05 & Xu et al. & $\begin{array}{l}\text { Alphaproteobacteria e } \\
\text { Gammaproteobacteria }\end{array}$ & Scopus \\
\hline
\end{tabular}

"Plastic biodegradation AND (Psychrophile OR Psychrophilic)" 


\begin{tabular}{|c|c|c|c|c|c|}
\hline 2011 & $\begin{array}{c}\text { JAMSTEC Rep Res } \\
\text { Dev }\end{array}$ & 3.2 & Sekiguchi et al. & $\begin{array}{c}\text { Shewanella, Moritella, } \\
\text { Psychrobacter e Pseudomonas }\end{array}$ & Google Scholar \\
\hline 2020 & Gene & 3.8 & Rogala et al. & $\begin{array}{c}\text { bactérias } \\
\text { produtoras de } \\
\text { PHA }\end{array}$ & Pubmed \\
\hline 2013 & $\begin{array}{l}\text { International } \\
\text { Biodeterioration \& } \\
\text { Biodegradation }\end{array}$ & 4.07 & $\begin{array}{l}\text { Singh } \\
\text { et al. }\end{array}$ & Pseudomonas sp. GBS.5 & Scopus \\
\hline 2010 & Water Research & 9.13 & $\begin{array}{l}\text { Trzcinski, \& } \\
\text { Stuckey }\end{array}$ & não revelado & $\begin{array}{c}\text { Google Scholar e } \\
\text { Scopus }\end{array}$ \\
\hline \multicolumn{6}{|c|}{ “PETases AND (bacteria OR fungus)" } \\
\hline 2019 & $\begin{array}{c}\text { Nature } \\
\text { Communications }\end{array}$ & 12.12 & Joo et al. & Ideonella sakaiensis 201-F6 & Google Scholar \\
\hline 2020 & $\begin{array}{c}\text { Process } \\
\text { Biochemistry }\end{array}$ & 2.95 & Costa et al. & Yarrowia lipolytica & Scopus \\
\hline 2020 & $\begin{array}{l}\text { Journal of } \\
\text { Environmental } \\
\text { Chemical } \\
\text { Engineering }\end{array}$ & 4.3 & $\begin{array}{l}\text { Kumar } \\
\text { et al. }\end{array}$ & $\begin{array}{l}\text { Rhococcus sp. } \\
\text { SSM1 }\end{array}$ & Scopus \\
\hline 2016 & $\begin{array}{c}\text { Process } \\
\text { Biochemistry }\end{array}$ & 2.95 & $\begin{array}{l}\text { Carniel } \\
\text { et al. }\end{array}$ & $\begin{array}{c}\text { Candida antarctica e Humicola } \\
\text { insolens }\end{array}$ & Scopus \\
\hline 2020 & $\begin{array}{l}\text { Frontiers in } \\
\text { Microbiology }\end{array}$ & 4.23 & Almeida et al. & Streptomyces sp. SM14 & Pubmed \\
\hline \multicolumn{6}{|c|}{ “Extremophile AND Plastic biodegradation" } \\
\hline 2018 & $\begin{array}{c}\text { International } \\
\text { Journal of } \\
\text { Advanced Scientific } \\
\text { Research and } \\
\text { Management }\end{array}$ & 7.58 & Rafiq et al. & $\begin{array}{l}\text { Halobacterium salinarium, } \\
\text { Halobacillus salinus, Vibrio } \\
\text { fischeri, Aeromonas spp. e } \\
\text { Staphylococcus epidermidis. }\end{array}$ & \\
\hline 2018 & RSC Advances & 3.1 & Chauhan et al. & $\begin{array}{l}\text { Exiguobacterium sibiricum } \mathrm{dr} 11 \\
\text { e Exiguobacterium undae } \mathrm{dr} 14\end{array}$ & \\
\hline 2011 & $\begin{array}{c}\text { Jamstec Rep Res } \\
\text { Dev }\end{array}$ & 3.2 & Sekiguchi et al. & $\begin{array}{c}\text { Shewanella, Moritella, } \\
\text { Psychrobacter e Pseudomonas }\end{array}$ & Google Scholar \\
\hline 2011 & $\begin{array}{c}\text { Applied } \\
\text { Microbiology and } \\
\text { Biotechnology }\end{array}$ & 3.5 & Dastgheib et al. & Marinobacter e Halomonas & \\
\hline 2018 & $\begin{array}{l}\text { Journal of Applied } \\
\text { Phycology }\end{array}$ & 3 & Ouada et al. & $\begin{array}{c}\text { Chlorophyta picocystis e } \\
\text { Chlorophyta graesiella }\end{array}$ & \\
\hline
\end{tabular}

Fonte: Autores.

Após uma análise mais acurada dos trabalhos encontrados, foi possível observar relatos da utilização de microrganismos degradadores de plásticos no ambiente marinho como os reportados por Sekiguchi et al., (2011) e Xu et al. (2019), com relatos da degradação de microplásticos (PCL) presentes no mar costeiro da China, por representantes dos grupos taxonômicos Alphaproteobacteria, Rhodobacteraceae e Gammaproteobacteria. Embora tenha sido utilizado o descritor "Plastic 
biodegradation AND cold oceans" para a recuperação destas informações, nenhum dos microrganismos comentados acima são psicrofílicos. Entretanto, a maioria dos microrganismos relatados nos trabalhos são de regiões que apresentam climas oscilantes, entre períodos de frio e calor durante o ano.

Para a utilização do descritor "Plastic biodegradation AND (Psychrophile OR Psychrophilic)", foi possível observar, que a maioria dos estudos estavam relacionados à utilização de microrganismos de ambientes frios em processos de biodigestão de diferentes tipos de resíduos, incluindo resíduos orgânicos, industriais, águas residuais e esterco, bem como diversos trabalhos relacionados à biorremediação do solo contaminado com petróleo, compostos orgânicos, poluentes clorados, produtos farmacêuticos, e outros. O resumo das aplicações mais relevantes encontradas estão ilustradas na Figura 2, sendo a biorremediação de outros compostos poluentes, a utilização mais observada nos trabalhos.

Figura 2 - Aplicações das pesquisas encontradas com a combinação "Plastic biodegradation AND (Psychrophile OR Psychrophilic)".

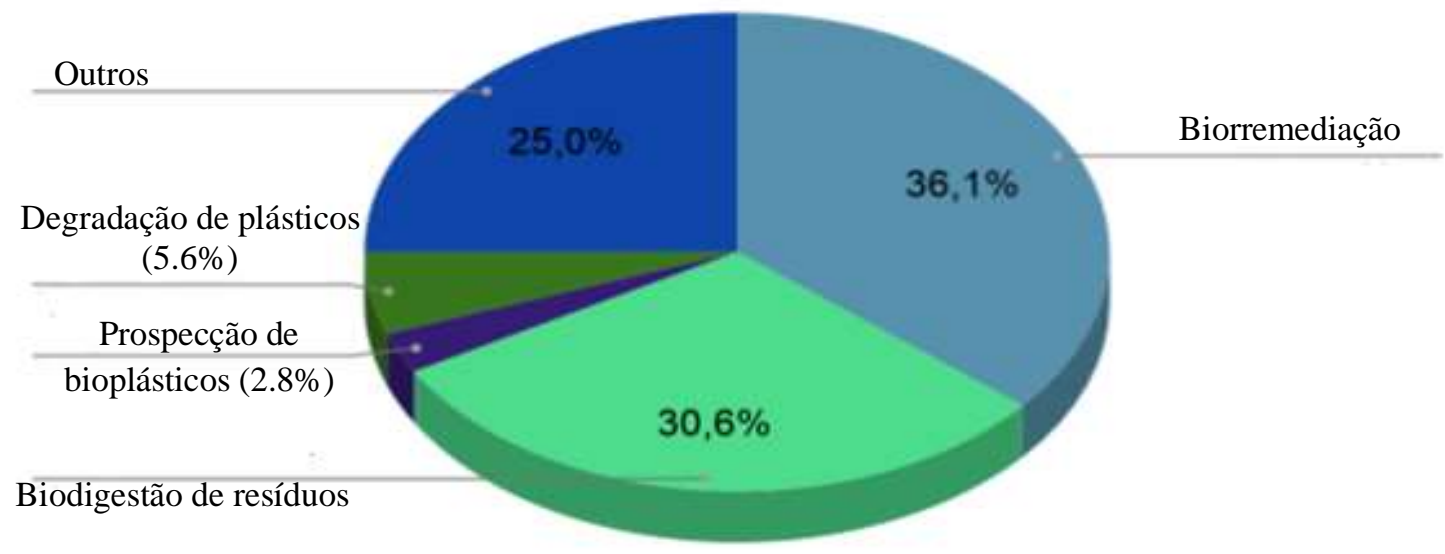

Fonte: Autores

Com relação a busca utilizando o descritor "PETases AND (bacteria OR fungus)", foi possível observar entre os 22 trabalhos selecionados, ensaios que utilizaram combinações de mais de um microrganismo para avaliar a hidrólise do PET. No trabalho de Carniel et al. (2016), os autores verificaram que os isolados Candida antarctica e Humicola insolens apresentaram potencial de hidrólise do PET, em sinergia onde, $H$. insolens não realizava a última etapa de reação da hidrólise sem a ação complementada pela levedura $C$. antarctica. Da mesma forma, Meyer-Cifuentes et al. (2020) testaram a ação sinérgica dos isolados Marinobacter, Pseudooceanicola e Saccharospirillum na degradação de amostras de PETs, encontrando resultados de hidrólise apenas na associação das linhagens. Estudos de combinações de microrganismo vêm se mostrando promissores pois certas linhagens são capazes de quebrar as ligações dos polímeros plásticos, porém não são capazes de assimilar e/ou degradar os polímeros, o que pode ser realizado por outras linhagens microbianas em associação. Todos estes estudos resultam importantes passos para futuros avanços na área de degradação do plástico, pois com a compreensão dos mecanismos, estruturas e funções biológicas das enzimas degradadoras de plásticos, será possível avançar a um caminho de aplicabilidade destes resultados.

Seguindo da mesma forma, após a realização da busca com o descritor "Extremophile AND plastic biodegradation", foi possível observar a carência de estudos relacionados à degradação dos plásticos com células microbianas extremófilas. A grande maioria dos trabalhos foi relacionada a pesquisas de diversas áreas como biorremediação, utilização de plantas 
extremófilas, produção de biogás, plásticos como vetor para disseminação de resistência a antibióticos, bem como trabalhos que focavam em pesquisas com o objetivo de melhorar a produção de compostos produzidos por microrganismos extremófilos, como o bioplástico polihidroxialcanoatos (PHAs) (Cantera et al. 2018; Pärnänen et al. 2014; Perérez et al. 2019).

Trabalhos unicamente focados na biodegradação de plásticos foram encontrados somente pela plataforma de busca Google Scholar, com pesquisas descrevendo microrganismos halofílicos e halotolerantes capazes de produzirem enzimas degradadoras de polímeros. Rafiq et al. (2018), avaliaram 11 linhagens, dentre elas, Halobacterium salinarium, Halobacillus salinus, Vibrio fischeri, Aeromonas spp. e Staphylococcus epidermidis, capazes de produzir enzimas degradadoras de polietileno de baixa densidade (LDPE). Chauhan et al. (2018) também avaliaram duas linhagens Exiguobacterium sibiricum DR11 e Exiguobacterium undae DR14, com potencial para biodegradação de poliestireno (PE). Neste estudo, E. undae pôde utilizar poliestireno como fonte de carbono. O trabalho de Sekiguchi e colaboradores (2011), foi o primeiro estudo a registrar bactérias degradadoras de policaprolactona (PCL) isoladas de profundidades marinhas $(5 \mathrm{~km})$. Os autores avaliaram linhagens microbianas em ensaios de degradação de polímeros plásticos, entretanto, obtiveram resultados apenas para a degradação do plástico biodegradável PCL, por linhagens dos gêneros Shewanella, Moritella, Psychrobacter e Pseudomonas. Por outro lado, de acordo com Urbanek et al., (2018), os microrganismos isolados de ambientes frios, capazes de produzir enzimas potencialmente aplicáveis para degradação de materiais plásticos como as lipases e que são mais frequentemente mencionados em trabalhos científicos são espécies pertencentes aos gêneros Pseudomonas, Streptomyces, Corynebacterium, Arthrobacter, Micrococcus e Rhodococcus.

Para entender as dificuldades dos trabalhos de biodegradação de plásticos, primeiramente temos que entender as principais limitações que se enfrenta na pesquisa. Em ambientes frios, assim como nas profundezas dos oceanos, as taxas de degradação podem ser ainda menores devido à baixa temperatura ambiente, baixo consumo de energia e ausência de luz solar (Bergmann \& Klages, 2012). Especificamente, a limitada exposição da luz ultravioleta pode ter o maior impacto, pois este cumpre um papel chave no início do processo oxidativo (O'Brine \& Thompson 2010). Além disso, o nível de oxigênio no fundo do mar pode ser muito baixo, tornando as atividades metabólicas oxidativas necessárias, improváveis de ocorrer sob essas condições.

Na atualidade, informações sobre a taxa de mineralização do plástico dentro dos oceanos são praticamente inexistentes. Porém, existem pequenos avanços que são de grande importância para futuros estudos, como os poços visualizados nos detritos de plástico com o formato das células bacterianas encontradas no ambiente marinho (Zettler et al., 2013) e o descobrimento de uma série de genes de degradação de xenobióticos presentes nas comunidades microbianas encontradas no biofilme formado ao redor dos plásticos, que estão provavelmente envolvidos na degradação do plástico (Bryant et al., 2016; Debroas et al., 2017; Dussud et al., 2018).

É necessário, uma compreensão ampla das vias metabólicas envolvidas no processo de biodegradação microbiana, ou seja, estudar vias co-metabólicas, já que a biodegradação microbiana pode envolver comunidades microbianas complexas (Syranidou et al., 2017). Além da necessidade de gerar protocolos padrões para testar a biodegradação de plásticos, uma vez que diversas metodologias são encontradas nos trabalhos atuais, tornando difícil a comparação estatística e realista dos resultados obtidos.

\section{Conclusão}

A poluição e os danos ambientais causados pelos plásticos têm aumentado a consciência e pesquisa para buscar soluções a esta problemática. Com o levantamento dos trabalhos apresentados na presente revisão, foi possível perceber que mesmo com muitos avanços na área, com a descoberta de linhagens degradadoras, ainda há um longo caminho por percorrer até alcançar a aplicabilidade de sistemas biológicos para tratar a contaminação oceânica por plásticos. Ainda há muito por compreender sobre 
vias metabólicas e genes envolvidos na biodegradação de polímeros plásticos por microrganismos de ambientes frios. O presente trabalho bibliográfico abre portas para realização de futuros estudos incluindo isolamento de microrganismos de ambientes frios, triagem funcional bem como a possibilidade da elaboração de novas abordagens para caracterizar os compostos poluidores e seus metabólitos, ampliando assim, as informações sobre a microbiota marinha psicrofílica e/ou psicrotolerante em metabolizar e/ou degradar polímeros plásticos.

\section{Agradecimentos}

Os autores agradecem à Universidade Federal da Integração Latino-Americana pelos recursos referentes ao Edital $N^{\circ}$ 105/2020/PRPPG - Programa Institucional Prioridade América-Latina e Caribe.

\section{Referências}

Almeida, E. L. Rincón, A. F. C. Jackson, S. A. \& Dobson, L. D. W. (2019). In silico Screening and Heterologous Expression of a Polyethylene Terephthalate Hydrolase (PETase)-Like Enzyme (SM14est) With Polycaprolactone (PCL)-Degrading Activity, From the Marine Sponge-Derived Strain Streptomyces sp. SM14. Front Microbiol, 10:2187.

Andrady, A. L. (2011). Microplastics in the marine environment. Marine pollution bulletin, 62(8), 1596-1605. https://doi.org/10.1016/j.marpolbul.2011.05.030. Bergmann, M. \& Klages, M. (2012). Increase of litter at the Arctic deep-sea observatory HAUSGARTEN. Mar Pollut Bull, 64(12):2734-2741

Bryant, J. A. Clemente, T. M. Viviani, D. A. Fong, A. A. Thomas, K. A., Kemp, P. et al. (2016). Diversity and activity of aommunities inhabiting plastic debris in the North Pacific gyre. mSystems 1:e0024-16.

Camargo, J. F. Farias, J. B. Paim, M. G. Lopes, B. Teixeira, L. G. Rosa, M. P. \& Contesini, E. A. (2020). Antimicrobianos utilizados no tratamento da pneumonia aspirativa em cães: revisão de literatura. Research Society and Development, 9(12):e11091210805.

Cantera, S., Sánchez-Andrea, I., Lebrero, R., García-Encina, P. A., Stams, Alfons J. M., Muñoz, R. (2018). Multi-production of high added market value metabolites from diluted methane emissions via methanotrophic extremophiles. Bioresource Technology.

Carniel, A. Valoni, E. Junior, J. N. Gomes, A. C. \& Castro, A. M. (2016). Lipase from Candida antarctica (CALB) and cutinase from Humicola insolens act synergistically for PET hydrolysis to terephthalic acid. Process Biochemistry, 56(A):84-90

Chauhan, D. Agrawal, G. Deshmukh, S. Roy, S. S. \& Priyadarshini, R. (2018). Biofilm formation by Exiguobacterium sp. DR11 and DR14 alter polystyrene surface properties and initiate biodegradation. RSC Advances, 8(66):37590-37599.

Cook, G. Teufel, A. Kalra, I. Li, E. Wang, X, et al. (2019). The Antarctic psychrophiles Chlamydomonas spp. UWO241 and ICE-MDV exhibit differential restructuring of photosystem I in response to iron. Photosynth Res, 141(2):209-228.

Costa, A. M. Lopes, V. R. O. Vidal, L. Nicaud, J.-M. Castro, A. M. \& Coelho, A. Z. (2020). Poly(ethylene terephthalate) (PET) degradation by Yarrowia lipolytica: Investigations on cell growth, enzyme production and monomers consumption. Process Biochemistry, 95:81-90.

Dastgheib, S. M. M. Amoozegar, M. A. Khajeh, K. Shavandi, M. \& Ventosa, A. (2012). Biodegradation of polycyclic aromatic hydrocarbons by a halophilic microbial consortium. Applied Microbiology and Biotechnology, 95(3):789-798

Debroas, D. Mone, A. \& Ter Halle, A. (2017). Plastics in the North Atlantic garbage patch: a boat-microbe for hitchhikers and plastic degraders. Sci Total Environ, 599:1222-1232.

Dussud, C. Hudec, C. George, M. Fabre, P. Higgs, P. et al. (2018). Colonization of Non-biodegradable and Biodegradable Plastics by Marine Microorganisms. Front Microbiol 9:1571.

Dussud, C. \& Ghiglione, J.-F. (2014). Bacterial degradation of synthetic plastics. In: Marine Litter in the Mediterranean and Black Seas, (ed. F. Briand), Paris: CIESM Publisher.

Ellen MacArthur Foundation (2016). The new plastics economy: rethinking the future of plastics. Recuperado de https://www.newplasticseconomy.org/about/publications/report-2016

Eriksen, M., Lebreton, L. C., Carson, H. S., Thiel, M., Moore, C. J., Borerro, J. C., \& Reisser, J. (2014). Plastic pollution in the world's oceans: more than 5 trillion plastic pieces weighing over 250,000 tons afloat at sea. PloS one, 9(12), e111913.

Geweret, B. Plassmann, M. M., \& MacLeod, M. (2015). Pathways for degradation of plastic polymers floating in the marine environment. Environ Sci Processes Impacts, 17:1513-1521.

Geyer, R. Jambeck, J. R. \& Law, K. L. (2017). Production, use, and fate of all plastics ever made. Sci Adv, 3(7), e1700782.

Giacomucci, L. Raddadi, N. Soccio, M. Lotti, N. \& Fava, F. (2020). Biodegradation of polyvinyl chloride plastic films by enriched anaerobic marine consortia. Marine Environmental Research, 158:104949. 
Gregory, M. R. (2009). Environmental implications of plastic debris in marine settings-entanglement, ingestion, smothering, hangers-on, hitch-hiking and alien invasions. Philos Trans $R$ Soc B, 364(1526), 2013-2025.

Haernvall, K. Zitzenbacher, S. Wallig, K. Yamamoto, M. Schick, M. B. Ribitsch, D. \& Guebitz, G. M. (2017). Hydrolysis of ionic phthalic acidbased polyesters by wastewater microorganisms and their enzymes. Environ Sci Technol, 51:4596-4605.

Haernvall, K. Zitzenbacher, S. Biundo, A. Yamamoto, M. Schick, M. B. Ribitsch, D. \& Guebitz, G.M. (2018). Enzymes as enhancers for the biodegradation of synthetic polymers in wastewater. ChemBioChem, 19:317-325.

Harshvardhan, K. \& Bhavanath, J. (2013). Biodegradation of low-density polyethylene by marine bacteria from pelagic waters, Arabian Sea, India. Marine Pollution Bulletin, 77(1-2):100-106.

Harrison, J. P. Sapp, M. Schratzberger, M. \& Osborn, A. M. (2011). Interactions between microorganisms and marine microplastics: a call for research. Mar Technol Soc J, 45:12-20.

Ingavale, R. \& Raut, P. D. (2018). Comparative biodegradation studies of LDPE and HDPE using Bacillus weihenstephanensis isolated from garbage soil. Nat Environ Pollut Technol, 17, 649-655.

İpekoglu, B. Böke, H. \& Çizer, Ö. (2007). Assessment of material use in relation to climate in historical buildings. Build Environ, $42: 970-978$.

Jacquin, C. J. Odobel, C. Pandin, C. Conan, P. Pujo-Pay, M. et al. (2019). Microbial Ecotoxicology of Marine Plastic Debris: A Review on Colonization and Biodegradation by the "Plastisphere". Front Microbiol, 10:865.

Joo, S. Cho, I. J. Seo, H. Son, H. F. Sagong, H.-Y. et al. (2018). Structural insight into molecular mechanism of poly (ethylene terephthalate) degradation. Nat Commun, 9(1):382.

Kumar, V. Maitra, S. S. Singh, R. \& Burnwal, D. K (2020). Acclimatization of a newly isolated bacteria in monomer tere-phthalic acid (TPA) may enable it to attack the polymer poly-ethylene tere-phthalate (PET). Journal of Environmental Chemical Engineering, 8(4):103977

Kumaravel, S. Hema, R. \& Lakshmi, R. (2010). Production of polyhydroxybutyrate (Bioplastic) and its biodegradation by Pseudomonas lemoignei and Aspergillus niger. E-J, Chem, 7:S536-S542.

Kyaw, B. M. Champakalakshmi, R. Sakharkar, M. K. Lim, C. S. \& Sakharkar, K. R. (2012). Biodegradation of low density polythene (LDPE) by Pseudomonas species. Indian J Microbiol, 52:411-419.

Lamendella, R. Strutt, S. Borglin, S. Chakraborty, R. Tas, N. et al. (2014). Assessment of the Deepwater Horizon oil spill impact on Gulf coast microbial communities. Front Microbiol, 5:130.

Margesin, R. \& Miteva, V. (2011). Diversity and ecology of psychrophilic microorganisms. Res Microbiol, 1623:46-361.

Mattsson, K. Hansson, L. A. \& Cedervall, T. (2015). Nano-plastics in the aquatic environment. Environ Sci Proces and Impacts, 17(10), 1-17

Martínez-Tobón, D. I. Gul, M. Elias, A. L. \& Sauvageau, D. (2018). Polyhydroxybutyrate (PHB) biodegradation using bacterial strains with demonstrated and predicted PHB depolymerase activity. Appl Microbiol Biotechnol, 102:8049-8067.

Mehetre, G. Shah, M. Dastager, S. G. \& Dharne, M. S. (2018). Untapped bacterial diversity and metabolic potential within Unke shwar hot springs, India. Arch Microbiol, 200:753-770.

Meyer-Cifuentes, I., Werner, J. Heuer, A. Jehmlich N. \& Öztürk, B. (2020). Synergistic degradation of a biodegradable plastic film by a marine microbial community. Access Microbiology, (2)7A.

Nimchua, T., Punnapayak, H., \& Zimmermann, W. (2007). Comparison of the hydrolysis of polyethylene terephthalate fibers by a hydrolase from Fusarium oxysporum LCH I and Fusarium solani f. sp. pisi. Biotechnol J, 2:361-364.

O'Brine, T., \& Thompson, R. C. (2010). Degradation of Plastic Carrier Bags in the Marine Environment. Marine Pollution Bulletin, 60, $2279-2283$.

Ottoni, J. R., Rodrigues, S. T., Maia, O. V., \& Passarini, M. R. Z. (2020). Characterization of amylase produced by cold-adapted bacteria from Antarctic samples. Biocatalysis and Agricultural Biotechnology, 20:101452.

Ouada, S. B. Ali, R. B. Leboulanger, C. Zaghden, H. Choura, S. Ouada, H. B. \& Sayadi, S. (2018). Effect and removal of bisphenol A by two extremophilic microalgal strains (Chlorophyta). Journal of Applied Phycology, 30:1765-1776

Pärnänen, K., Karkman, A., Virta, M., Eronen-Rasimus, E., \& Kaartokallio, H. (2015). Discovery of bacterial polyhydroxyalkanoate synthase (PhaC)-encoding genes from seasonal Baltic Sea ice and cold estuarine waters. Extremophiles, 19(1), 197-206.

Paço, A., Duarte, K. da Costa, J. P., Santos, P. S. M., Pereira, R., Pereira, M. E., et al. (2017). Biodegradation of polyethylene microplastics by the marine fungus Zalerion maritimum. Science of The Total Environment, 586:10-15.

Peixoto, J., Silva, L. P., \& Krüger, R. H. (2017). Brazilian Cerrado soil reveals an untapped microbial potential for unpretreated polyethylene biodegradation. $J$ Hazard Mater, 324,634-644.

Pereira, A. S., Shitsuka, D. M., Parreira, F. J., \& Shitsuka, R. (2018). Metodologia da pesquisa científica. UFSM. https://repositorio.ufsm.br/bitstream/há ndle/1/15824/Lic_Computacao_Metodologia-Pesquisa-Cientifica.pdf?sequence=1

Pérez, R., Cantera, S., Bordel, S., García-Encina, P. A., \& Muñoz, R. (2019). The effect of temperature during culture enrichment on methanotrophic polyhydroxyalkanoate production. International Biodeterioration \& Biodegradation. 
Pramila, R. (2012). Brevibacillus parabrevis, Acinetobacter baumannii and Pseudomonas citronellolis - Potential candidates for biodegradation of low density olyethylene (LDPE). J Bacterio. Res, 4:9-14.

Ritchie, H., \& Roser, M. (2018). Plastic pollution. https://ourworldindata.org/plastic-pollution.

Rogala, M. M. Gawor, J. Gromadka, R., Kowalczyk, M., \& Grzesiak, J. (2020). Biodiversity and Habitats of Polar Region Polyhydroxyalkanoic Acid-Producing Bacteria: Bioprospection by Popular Screening Methods. Genes, 11(8):873.

Sekiguchi, T. Sato, T, Enoki, M. Kanehiro, H. Uemtsu, K. \& Kato, C. (2010). Isolation and characterization of biodegradable plastic degrading bacteria from deep-sea environments. JAMSTEC Rep Res Dev, 11:33-41

Sekiguchi, T., Sato, T., Enoki, M., Kanehiro, H. \& Kato, C. (2010) Procedure for isolation of the plastic degrading piezophilic bacteria from deep-sea environments. Journal of Japanese Society for Extremophiles, 9(1):25-30.

Sheik, S. Chandrashekar, K. R. Swaroop, K. \& Somashekarappa, H. M. (2015). Biodegradation of gamma irradiated low density polyethylene and polypropylene by endophytic fungi. Int Biodeterior Biodegradation, 105, 21-29.

Singh, G. B. Gupta, S., \& Gupta, N. (2013). Carbazole degradation and biosurfactant production by newly isolated Pseudomonas sp. strain GBS.5. International Biodeterioration \& Biodegradation, 84:35-43

Sowmya, H. V. Ramalingappa, M. \& Krishnappa, M. (2012). Degradation of polyethylene by Chaetomium sp. and Aspergillus Flavus. Int J Recent Sci Res, 3:513-517.

Summera Rafiq, R., Ishrath Razia, S. K., Jasmine Shahina, K., \& Vijaya, R. (2018). Screening of Extracellular Hydrolytic Enzymes from Halophilic bacteria and Biodegradation of LDPE. International Journal of Advanced Scientific Research and Management, 3(7):195-200.

Syranidou, E., et al (2017). Biodegradation of weathered polystyrene films in seawater microcosms. Scientific Reports.

Trzcinski, A. P., \& Stuckey, D. C. (2010). Treatment of municipal solid waste leachate using a submerged anaerobic membrane bioreactor at mesophilic and psychrophilic temperatures: Analysis of recalcitrants in the permeate using GC-MS. Water Research, 44(3): 671-680

Undabarrena, A., Beltrametti, F., Claverías, F. P., González, M. Moore, E. R. B., \& Seeger, M. (2016). Cámara B. Exploring the diversity and antimicrobial potential of marine actinobacteria from the comau fjord in Northern Patagonia, Chile. Front Microbiol, 7:1135.

Urbanek, A., K. Rymowicz, W., \& Mirończuk, M. A. (2018). Degradation of plastics and plastic-degrading bacteria in cold marine habitats. Applied Microbiology and Biotechnology, 102:7669-7678.

Veiga, J. M., Fleet, D., Kinsey, S., Nilsson, P., Vlachogianni, T. et al. (2016). Identifying sources of marine litter. MSFD GES TG Marine Litter Thematic Report, JRC Technical Report, EUR 28309

Waller, C. L. Griffiths, H. J. Waluda, C. M. Thorpe, S. E. Loaiza, I. et al. (2017). Microplastics in the Antarctic marine sy stem: an emerging area of research. Sci Total Environ 598:220-227.

Xu, X., Wang, S., Gao, F., Li, J., Zheng, L. et al. (2019). Marine microplastic-associated bacterial community succession in response to geography, exposure time, and plastic type in China's coastal seawaters. Marine Pollution Bulletin, 145: 278-286.

Yang, M., Dongwei, Lu., Jiaxuan, Y., Yumeng, Z., Qi, Z. et al. (2019). Carbon and nitrogen metabolic pathways and interaction of cold-resistant heterotrophic nitrifying bacteria under aerobic and anaerobic conditions. Chemosphere, 234:162-170.

Yang, J., Yang, Y. Wu, W.,-M. Zhao, J. \& Jiang, L. (2014). Evidence of polyethylene biodegradation by bacterial strains from the guts of plastic-eating waxworms. Environ Sci Technol, 48, 13776-13784.

Yogabaanu, U,. Weber, J. F. F., Convey, P., Rizman-Idid, M., \& Alias, S. A. (2017). Antimicrobial properties and the influence of temperature on secondary metabolite production in cold environment soil fungi. Polar Sci, 14:60-67.

Zettler, E. R., Mincer, T. J., \& Amaral-Zettler, L. A. (2013). Life in the "plastisphere": microbial communities on plastic marine debris. Environ Sci Technol, 47:7137-7146. 